
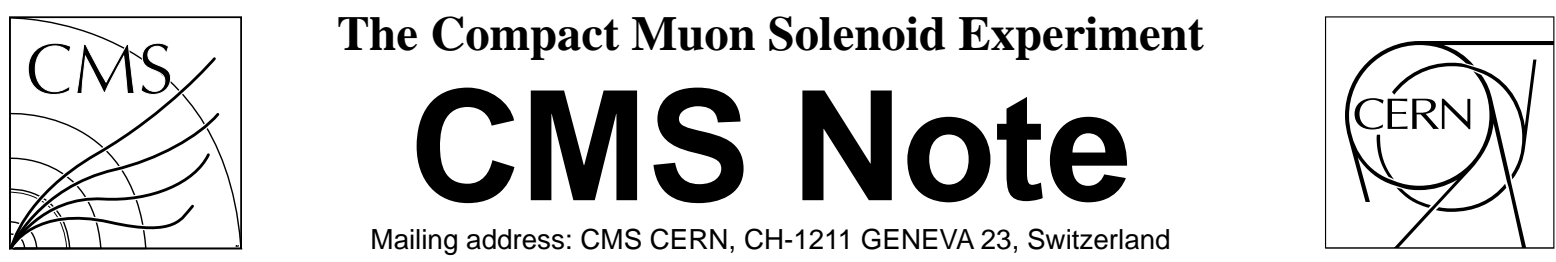

June 9, 2006

\title{
Detection of heavy Majorana neutrinos and right-handed bosons
}

\author{
S.N. Gninenko, M.M. Kirsanov, N.V. Krasnikov, V.A. Matveev \\ INR, Moscow, 117312, Russia
}

\begin{abstract}
The $S U_{C}(3) \otimes S U_{L}(2) \otimes S U_{R}(2) \otimes U(1)$ left-right (LR) symmetric model explains the origin of the parity violation in weak interactions and predicts the existence of additional $W_{R}$ and $Z^{\prime}$ gauge bosons. In addition, heavy right-handed Majorana neutrino states $N$ arise naturally within the LR symmetric model. The $N$ s could be partners of light neutrino states, related to their non-zero masses through the see-saw mechanism. This makes the searches of $W_{R}, Z^{\prime}$ and $N$ interesting and important.

This note describes a study of the potential of the CMS experiment to observe signals from the $N$ and $W_{R}$ production at the LHC. It is shown that their decay signals can be identified with a small background. For the integral LHC luminosity of $L_{t}=30 \mathrm{fb}^{-1}$, the $5 \sigma$ discovery of $W_{R}$ - boson and heavy Majorana neutrinos $N_{e}$ with masses up to $3.5 \mathrm{TeV}$ and $2.3 \mathrm{TeV}$, respectively is found possible.
\end{abstract}




\section{Introduction}

Among several extensions of the Standard Model (SM) that can be tested at LHC, see e.g. [1], the left-right (LR) symmetric model $S U_{C}(3) \otimes S U_{L}(2) \otimes S U_{R}(2) \otimes U(1)$ [2] is certainly one of the most interesting. The model embeds the $\mathrm{SM}$ at the scale of the order of $1 \mathrm{TeV}$ and naturally explains the parity violation in weak interactions as a result of the spontaneously broken parity. The model necessarily incorporates three additional gauge bosons $W_{R}$ and $Z^{\prime}$ and the heavy right-handed Majorana neutrino states $N$. The $N$ s can be the partners $\left(N_{l}\right)$ of the light neutrino states $\nu_{l}(l=e, \mu, \tau)$ and can provide their non-zero masses through the see-saw mechanism [3]. This makes LR model very attractive, since recent results from atmospheric, solar and reactor neutrino experiments confirm the existence of neutrino oscillations, see e.g. [4], and therefore provide strong evidence that neutrinos are not massless. The above mentioned experiments, though very impressive, provide no hints neither on the nature of the masses (i.e. they cannot distinguish Dirac and Majorana neutrinos) nor on their values. The only observation that can be used to give preference to some models compared to others is the fact that the neutrino masses are very small compared to the charged lepton and quark masses. On the contrary, experiments searching for signals from $N$ s with masses in the LHC energy range look quite promising, since the large number of extensions of the LR model predict the heavy neutrino masses to be somewhere between several hundred $\mathrm{GeV}$ and a few $\mathrm{TeV}$ [1].

There are several recent papers devoted to the study of the production of heavy neutrinos and new gauge bosons at $\mathrm{TeV}$ energy scale, see e.g. Ref. [5]- [9]. The experimentally interesting large magnetic moments of muon and tau neutrinos [10] associated with Majorana neutrino in the LR symmetric model are reported in [11]-[13]. In this paper we discuss the production and the experimental signature of heavy Majorana neutrinos and the associated heavy gauge bosons in the CMS detector at LHC. We perform our study in the framework of the minimal LR symmetric model [2]. The goal of the work is to examine how the direct production of $W_{R}$ and $N_{l}$ can be manifested at the LHC energy. The preliminary results on this study has been reported in [14]. We also discuss the optimal conditions for the observation of $W_{R}$ and $N$ signals in CMS at low luminosity taking into account all most important background sources.

The rest of the paper is organized as follows. In Section 2 we briefly remind the essence of the LR model and some constraints on its parameters. In Sections 3 the production and decays of $W_{R}$ and $N_{l}$ are considered. The description of the CMS detector simulation and reconstruction tools, and candidate events selection are presented in Sections 4 and 5, respectively. The main background sources are discussed in Section 6. In Section 7 we present the results of our analysis and discuss the CMS potential to discover $W_{R}$ and $N_{l}$. Section 7 contains concluding remarks.

\section{Left-right symmetric models}

LR symmetric models of electroweak interactions were proposed to explain the origin of parity non-conservation in weak interactions [2]. It is well known that in the SM gauge symmetry is broken spontaneously, while parity (and charge) conjugation is broken explicitly in the Lagrangian. In the LR symmetric model parity, on the contrary, is conserved in the Lagrangian and broken spontaneously together with the gauge symmetry.

An important question for the phenomenology of these models is the scale of the parity breaking. During a long time there has been an interest in models where the masses of the right handed $W_{R}$ and $Z$ ' are in the multi-TeV region. With the recent discovery of non-zero neutrino masses, the case for left-right models has become more compelling for two reasons: i) the right handed neutrino, which is necessary to implement the see-saw mechanism, is an integral part of these models and ii) the local $B-L$ symmetry which protects the right handed neutrino mass from being at the Plank scale is also part of the gauge symmetry.

In the minimal $S U(2)_{L} \times S U(2)_{R} \times U(1)_{B-L}$ model each generation of quarks and leptons carry the quantum numbers $Q_{L} \sim(1 / 2,0,1 / 3), Q_{R} \sim(0,1 / 2,1 / 3), L_{L} \sim(1 / 2,0,-1), L_{R} \sim(0,1 / 2,-1)$. The right-handed fields are doublets under $S U(2)_{R}$ and a right-handed neutrino $N_{R}$ should exist. The minimal Higgs sector consists of a bi-doublet $\phi \sim(1 / 2,1 / 2,0)$ and two triplets $\Delta_{R} \sim(1,0,2), \Delta_{L} \sim(0,1,2)$. After the spontaneous symmetry breaking, the phenomenological requirement $\left|v_{L}\right| \gg\left|k_{1}\right|,\left|k_{2}\right| \ll\left|v_{R}\right|$ for the vacuum expectation values $v_{L, R}$ and $k_{1,2}$ of the doublet and triplet Higgs fields ensures the suppression of the right-handed currents and the smallness of the neutrino mass.

The $S U(2)_{L} \times S U(2)_{R} \times U(1)_{B-L}$ gauge symmetry group implies that the usual left-handed gauge bosons $W_{L}^{i}(i=1,2,3)$, their right-handed counterparts $W_{R}^{i}$ and the $U(1)$ gauge boson $Y$ combine to form the physical charged and neutral gauge bosons and the photon. In general, the strength of the gauge interactions of these bosons is described by the coupling constants $g_{L}, g_{R}$ and $g^{\prime}$, respectively. However, strict LR symmetry $\Phi_{L} \leftrightarrow$ 
$\Phi_{R}, \Delta_{L} \leftrightarrow \Delta_{R}, \phi \leftrightarrow \phi+$, where $\Phi$ denotes fermions, leads to the relation $g_{L}=g_{R}$ at $M_{W_{R}}$, which will be assumed throughout this paper.

The weak eigenstates $W_{L}^{ \pm}$and $W_{R}^{ \pm}$mix in the mass eigenstates $W^{ \pm}$and $W^{\prime \pm}$. Assuming CP invariance, the mixing matrix is defined by the angle $\xi_{W}$ :

$$
\begin{gathered}
W^{ \pm}=\cos \xi_{W} W_{L}^{ \pm}+\sin \xi_{W} W_{R}^{ \pm} \\
W^{\prime \pm}=-\sin \xi_{W} W_{L}^{ \pm}+\cos \xi_{W} W_{R}^{ \pm}
\end{gathered}
$$

The weak eigenstate $W_{L}$ can be identified with the pure SM gauge boson. Similarly the neutrino mass eigenstates are mixtures of the weak eigenstates, parametrized by the angle $\xi_{N}$ :

$$
\begin{gathered}
\nu=\cos \xi_{N} \nu^{\prime}+\sin \xi_{N} N^{\prime} \\
N=-\sin \xi_{N} \nu^{\prime}+\cos \xi_{N} N^{\prime}
\end{gathered}
$$

Here, $\nu$ and $N$ are the light and heavy neutrino mass eigenstates, and $\nu^{\prime}=\nu_{L}+\nu_{L}^{c}$ and $N^{\prime}=\nu_{R}+\nu_{R}^{c}$ are the usual self-conjugate spinors. For simplicity, we do not take into account possible mixing between generations.

The charge-current interactions vertices for the left-chiral and right-chiral currents are given by

$$
\begin{gathered}
<\nu_{L}\left|W_{L}\right| e^{-}>=g / 2 \sqrt{2} W_{L}^{+\mu} \bar{\nu}_{L} \gamma_{\mu}\left(1-\gamma_{5}\right) e \\
<N_{R}\left|W_{R}\right| e^{-}>=g / 2 \sqrt{2} W_{R}^{+\mu} \bar{N}_{R} \gamma_{\mu}\left(1+\gamma_{5}\right) e
\end{gathered}
$$

The charge-current interactions for the mixed mass eigenstates can be obtained from these matrix elements.

The neutral gauge bosons in L-R models are mixtures of $W_{L, R}^{3}$ and $Y$. The mixing between the massive neutral bosons can be parametrized as

$$
\begin{gathered}
Z=\cos \xi_{Z} Z_{1}+\sin \xi_{Z} Z_{2} \\
Z^{\prime}=-\sin \xi_{Z} Z_{1}+\cos \xi_{Z} Z_{2}
\end{gathered}
$$

where $Z$ and $Z^{\prime}$ denote the mass eigenstates, and $Z_{1}$ and $Z_{2}$ denote the weak eigenstates of the massive neutral bosons. The field $Z$ can be identified as the corresponding SM boson.

The tree-level neutral current interaction for the physical $Z, Z^{\prime}$ is of the from

$$
L_{N C}=g / 2 \cos \Theta_{W}\left[\bar{f} \gamma_{\mu}\left(g_{V}^{f}-g_{V}^{f} \gamma_{5}\right) f Z_{\mu}+\bar{f} \gamma_{\mu}\left(g_{V}^{\prime f}-g_{V}^{\prime} \gamma_{5}\right) f Z_{\mu}^{\prime}\right.
$$

where

$$
\begin{gathered}
g_{V}^{f}=\cos \xi_{Z} g_{V}^{0 f}+\sin \xi_{Z} g_{V}^{\prime f}, \\
g_{A}^{f}=\cos \xi_{Z} g_{A}^{0 f}+\sin \xi_{Z} g_{A}^{\prime f}
\end{gathered}
$$

and 


$$
\begin{gathered}
g_{V}^{0 f}=I_{3}^{f}-2 Q^{f} \sin ^{2} \Theta_{W}, g_{V}^{\prime f}=\sqrt{\cos 2 \Theta_{W}} g_{V}^{0 f} \\
g_{A}^{0 f}=I_{3}^{f}, g_{A}^{\prime f}=-\sqrt{\cos 2 \Theta_{W}} g_{A}^{0 f}
\end{gathered}
$$

Here we take into account that in the LR model $I_{3 R}^{f}=I_{3 L}^{f} \equiv I_{3}^{f}$ for the third components of the $L / R$ isospin for a given fermion flavor $f$.

Existing experimental data constrain the $Z^{\prime}$ mass to the values $O(1) \mathrm{TeV}$ and the mixing among the neutral gauge bosons to the values below $O\left(10^{-4}\right)[15,16]$.

The lower bound on the $W^{\prime}$ mass derived from the $K_{L}-K_{S}$ mass difference is quite stringent, $M_{W^{\prime}} \gtrsim 1.6 \mathrm{TeV}$ [19], however with some uncertainties from the low energy QCD corrections to the kaon system. The bound on the mixing angle is as low as $\xi_{W} \lesssim 0.013$ [20]. The direct searches for $W^{\prime}$ at the Tevatron yield bounds $M_{W^{\prime}} \gtrsim 786$ $\mathrm{GeV}$ assuming a light (keV-range) $N$, and $M_{W^{\prime}} \gtrsim 650 \mathrm{GeV}$ assuming $M_{N}<M_{W^{\prime}} / 2$ [21]. These bounds are less stringent in more general LR models [22]. Effects of the lepton flavor violation induced by heavy Majorana neutrinos and some recent limits are discussed in [17] and [18], respectively.

The least tested components of the LR model are the masses and mixing of neutrinos. The analysis of the precision data constrains fermion mixing [23]: the 90\% CL bound for the electron neutrinos is $\left|\xi_{N}\right| \lesssim 0.081$.

The mixing angle $\xi_{W}$ is independent of the neutral current parameters. The value of $\xi_{N}$ depends rather strongly on the parameters of the model, allowing mixing up to $\xi \simeq 0.1$ [24].

\section{Heavy Majorana neutrino production and decay}

Among several reactions of $N$ and $W_{R}$ production in $p p$ collisions the most interesting are: i) $p+p \rightarrow 2 l^{ \pm}+X$ due to the $W_{R}$ fusion mechanism, ii) $p+p \rightarrow W_{R}+X \rightarrow N_{l}+l+X$ and iii) $p+p \rightarrow 2 N_{l}+X$ with the subsequent decay of $N_{l}$ 's into charged leptons $l$ and jets. The first reaction is quite similar to the process of lepton number violation in the double- $\beta$ decay and has a clear experimental signature as an advantage. However, its cross-section is small compared to the processes ii) and iii) [5]. The corresponding Feynman diagrams for processes $p p \rightarrow W_{R} \rightarrow l+N_{l}+X$, and $p p \rightarrow Z^{\prime} \rightarrow N_{l}+N_{l}+X$ followed by the decay $N_{l} \rightarrow l+j_{1}+j_{2}$ are shown in Figure 1.

The cross sections of these reactions depend on the following parameters:

- the value of the coupling constant $g_{R}$,

- the masses $M_{W_{R}}$ and $M_{N}$

- parameters of the CKM mixing matrix for the right-handed sector,

- the $W_{R}-W_{L}$ and $Z^{\prime}-Z$ mixing strengths, and

- the masses of the partners $N_{l}$ of the light neutrino state.

To simplify our study, we will use the following standard assumptions:

- the mixing angles are small

- right-handed CKM matrix is identical to the left-handed one, and

- $g_{R}=g_{L}$

Under these conditions and given the Higgs sector with Majorana masses for neutrinos, $Z^{\prime}$ is about 1.7 times heavier than $W_{R}[5,6]$. For this reason the process going through $Z^{\prime}$ has smaller cross section. In addition, its signature is more complicated. Thus, we focus our study on the process ii) going through the direct production of $W_{R}$. In addition, we assume that only the mass $M_{N_{e}}$ of the heavy neutrino predominantly coupled to the electron flavor is accessible at LHC, other $N_{l}$ with masses being very large are not produced. As shown in Figure 1 the 
right-handed Majorana neutrino decays into a charged lepton $l^{ \pm}$and an off-shell $W_{R}$ boson which decays into a pair of quarks (jets $(j)$ after hadronisation). This results in a final state $l l j j$. Assuming the electron decay channel of $N_{e}$ results in the $e_{1} e_{2} j_{1} j_{2}$ final states:

$$
N_{e} \rightarrow e W_{R}^{*} \rightarrow e+2 j e t s
$$

The case with degenerated masses of $N_{l}$ differs by the existence of reactions with muons and $\tau$ and by the additional factor in the cross section of the reaction $p p \rightarrow W_{R} \rightarrow e+N_{e}+X$ due to slightly different $W_{R}$ branching ratio. This factor differs from unity by not more than $20 \%$ (see below Figure 3 ). Thus, one can consider only two variables, namely $M_{W_{R}}$ and $M_{N_{e}}$ that could vary independently. The point $M_{N_{e}}=500 \mathrm{GeV}$ and $M_{W_{R}}=2000$ $\mathrm{GeV}$ in this parameter space we will call below a reference point (LRRP).

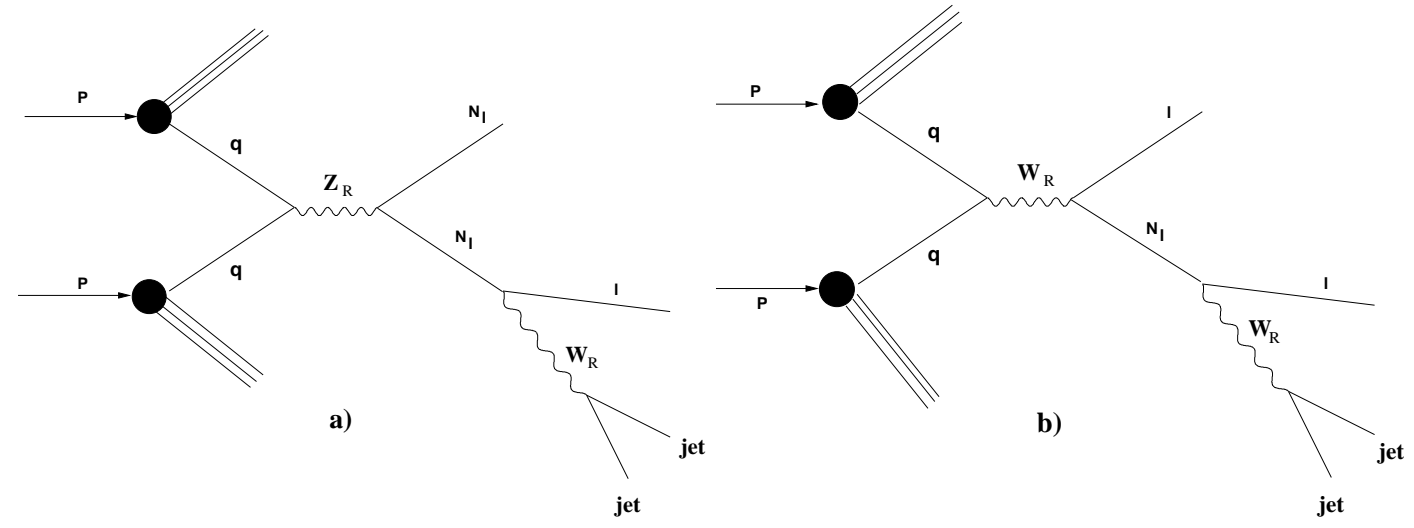

Figure 1: Feynman diagrams for production of the heavy neutrino through a) $Z^{\prime}$ bosons, b) $W_{R}$ bosons.

For the signal event generation and calculation of cross sections PYTHIA 6.2 [25] is used with default CTEQ5L parton distribution functions of ref. [26]. The code includes the LR symmetric model with the standard assumptions mentioned above.

The dependence of the $W_{R}$ total production cross section on its mass is shown in Figure 2 . The fraction $W_{R}^{+} /\left(W_{R}^{+}+\right.$ $\left.W_{R}^{-}\right)$for $W_{R}$ produced in pp collisions depends on $M_{W_{R}}$ and changes from $\simeq 70 \%$ at $M_{W_{R}} \simeq 1 \mathrm{TeV}$ to $\simeq 95 \%$ at $M_{W_{R}} \simeq 10 \mathrm{TeV}$. Thus, at higher $W_{R}$ boson masses the production of $W_{R}^{+}$boson dominates. Since the $W_{R}$ with mass below $1 \mathrm{TeV}$ is excluded by indirect analysis [6], the mass region above $1 \mathrm{TeV}$ is studied in this note.

In Figure 3 the signal cross section defined as the product of the total $W_{R}$ production cross section and the branching ratio of $W_{R}$ decay into electron and $N_{e}$ is shown as a function of $M_{N_{e}}$ mass for different masses of $W_{R}$. The production cross-section for $p p \rightarrow W_{R} \rightarrow e N_{e}$ was found to be at least one order of magnitude higher than that for the $p p \rightarrow Z^{\prime} \rightarrow N_{e} N_{e}$ process. Therefore, the channel $p p \rightarrow W_{R} \rightarrow e N_{e}$ is the most suitable for the heavy neutrino search.

\section{The detector simulation and reconstruction}

The detection of $W_{R}, N_{e}$ signals was studied using the full CMS detector simulation and reconstruction chain.

The main features of this analysis are listed below. If not specified otherwise, the numbers refer to LRRP.

- electron triggers were analysed. Events were reconstructed and further analysed only if there was a default L1 (level 1) trigger (single or double electron). The efficiency of this trigger on the events with both electrons in the ECAL (Electromagnetic Calorimeter) acceptance and with $E_{t}>20 \mathrm{GeV}$ (only such events were passed to the full detector simulation program) is compatible with $100 \%$. The efficiency of HLT (high level trigger) (single or double electrons) for the same events is $99 \%$.

- $E_{t}$ cut on electrons (and positrons, below, for simplicity, both are called electrons) was $20 \mathrm{GeV}$.

- it was required that electrons should be isolated in the Tracker. This was found to be very important. The full simulation shows that it is not possible to use non-isolated electrons (as one could try to do in the simplified fast simulation) since they are found in many jets. The isolation of electrons in the Tracker is determined by 


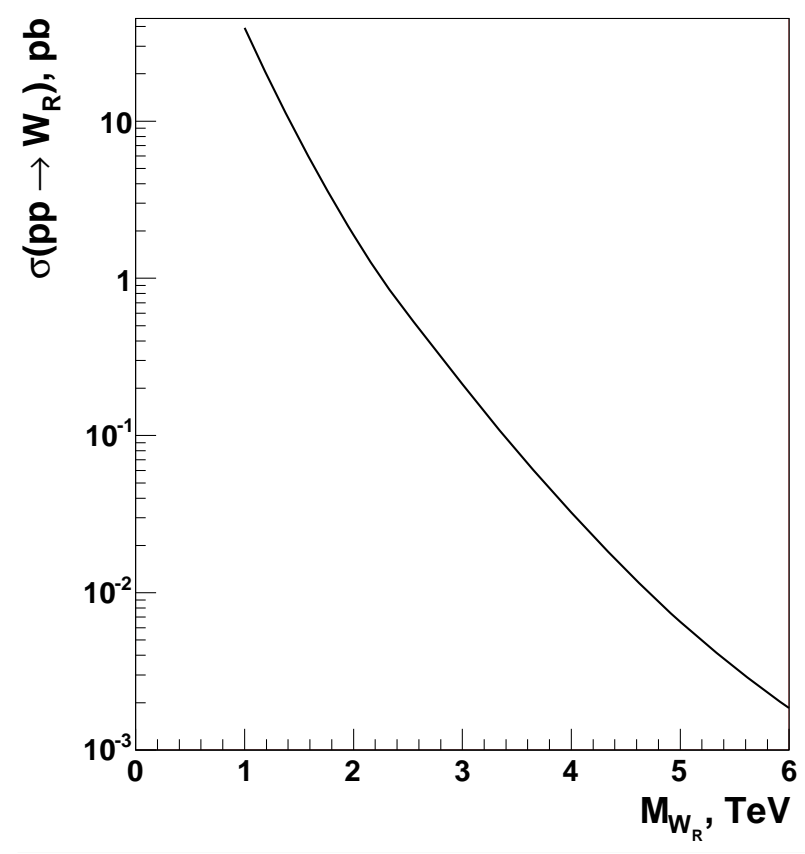

Figure 2: The dependence of the cross section $\sigma\left(p p \rightarrow W_{R}\right)$ on the $W_{R}$ mass

using the following criterion: not more than one charged particle with $P_{t}>2 G e V$ in the cone with a radius of 0.3 around the electron track.

- if the reconstructed electron was in a cone of radius 0.05 around some MC electron and its energy is smaller that $2 E_{M C}$, they are called matched. Using this criterion, we measured the following characteristics of the electron reconstruction and our electron selection: reconstruction efficiency (including isolation cuts) 86\%; efficiency of the isolation cuts to the electrons matched to MC 91\%; purity of the electron sample before isolation cuts $60 \%$; purity of the electron sample after isolation cuts $90.4 \%$.

- jets were reconstructed by the Iterative Cone algorithm with cone radius $\mathrm{R}=0.5$. The algorithm for the correction of the energy measurement that takes into account different response of calorimeters to electrons (gammas) and hadrons was used. The $E_{t}$ cut on corrected jets was $40 \mathrm{GeV}$.

\section{Selection of candidate events and the analysis variables}

The signal event candidates were preselected by requiring at least four reconstructed objects (criteria above) in the final state (below this is called primary selection):

- two or more isolated electrons;

- at least two jets.

The two jets with the maximal transverse momentum were used as a signal jet pair $j_{1}, j_{2}$. We found that in more than $90 \%$ of signal events we choose the correct jets from the heavy neutrino decay if these jets are reconstructed ${ }^{1)}$.

The following cuts and variables have been used in the further analysis:

- events with only two isolated electrons $e_{1}, e_{2}$ were selected. The invariant mass of this lepton pair $M_{e e}$ was required to be greater than $200 \mathrm{GeV}$.

- $M_{N_{e}}^{c a n d}$ - the invariant mass of an electron and two selected jets. Since there are two electrons selected, both combinations, $e_{1} j_{1} j_{2}$ and $e_{2} j_{1} j_{2}$ were considered. This variable was required to be inside some window

1) Correct means that the jet is inside a cone with radius 0.05 around the quark of the PYTHIA event record produced in the decay of the heavy neutrino 


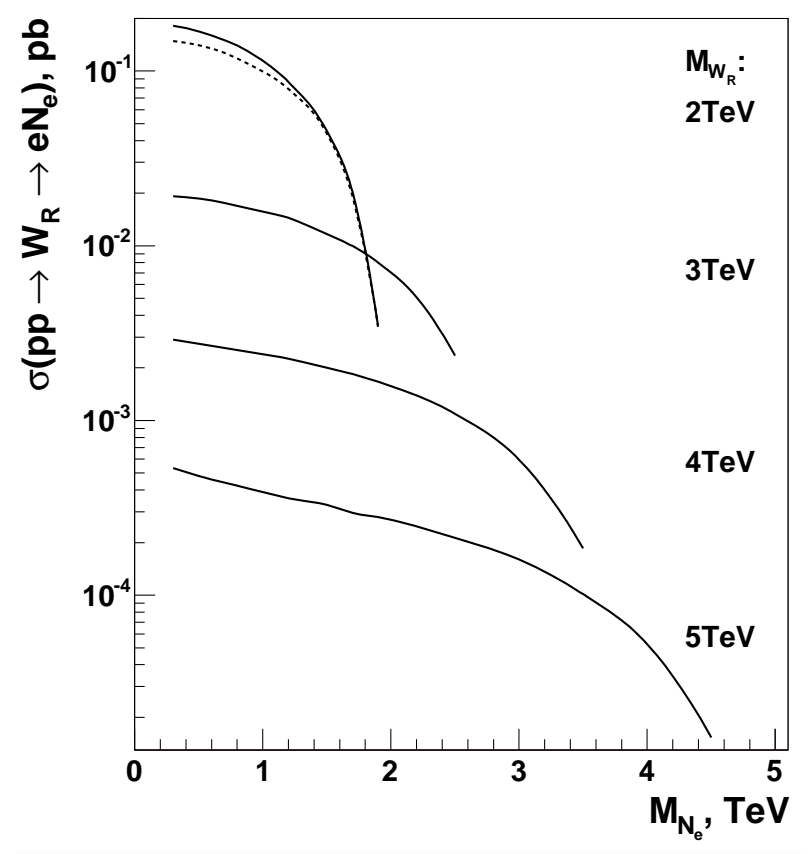

Figure 3: The dependence of the value $\sigma\left(p p \rightarrow W_{R}\right) \cdot \operatorname{Br}\left(W_{R} \rightarrow e^{ \pm} N_{e}\right)$ on the heavy neutrino mass. For the mass $M_{W_{R}}=2 \mathrm{TeV}$, the dashed line illustrates the dependence of the same value for the case of degenerated $N_{l}$ masses.

with the width optimized to obtain the highest sensitivity in presence of background. The full width of this mass window at LRRP is $110 \mathrm{GeV}$.

- $M_{W_{R}}^{c a n d}$ - the invariant mass of the combination $e_{1} e_{2} j_{1} j_{2}$ of two selected electrons and jets.

In Figure 4 the distribution of the reconstructed mass $M_{N_{e}}^{c a n d}$ is shown for events simulated at LRRP. The reason for this distribution to be non symmetric is two-fold. The additional broad peak or long tail above the heavy neutrino mass is due to the wrong choice of lepton. The tail toward zero in the $M_{N_{e}}^{c a n d}$ distribution is due to the emission of gluons and to the heavy neutrino decay into $t$ quarks $\left(N_{e} \rightarrow e W_{R} \rightarrow e t b\right)$ that produce wide jets or several jets. The distribution of the $M_{W_{R}}^{c a n d}$ mass for the same reconstructed events is shown in Figure 5.

In Figure 6 the efficiency of the primary selection and the efficiency of reconstruction of $W_{R}$ decay products are shown for the case of $M_{W_{R}}=2 \mathrm{TeV}$. One can see that for neutrino masses with $r=M_{N_{e}} / M_{W_{R}} \lesssim 0.3$ the reconstruction efficiency drops. The reason is that the heavy neutrino decay products are too close to each other in the $\eta-\phi$ space. As a result, two jets are sometimes reconstructed as a single jet or an electron candidate does not pass the isolation criteria.

\section{The background}

Background is expected from the SM processes with a lepton pair and at least two jets in the final state.

To evaluate the amount of background, these events were simulated with PYTHIA and processed by the same reconstruction program and passed the same selection criteria as signal events. The initial numbers of background events (see below Table 1) correspond to the statistics that would be collected at the CMS detector for the integrated luminosity $L_{t}=30 \mathrm{fb}^{-1}$. The following sources of background events are considered:

- the first background that was studied was the $Z W$ process. This background was important in the heavy neutrino search at LEP [27]. It was simulated with lepton decay modes of $W$ and hadron decay modes of $Z$ forbidden. The cross section of this process is of the order of the signal cross section at LRRP. Due to the fact that the tail of the $Z$ mass distribution is rather long and that the signal events usually have a large value of $M_{l l}$ a lower cut $M_{l l}>200 \mathrm{GeV}$, well above the $Z$ mass central value (see Figure 7), has been used to suppress this kind of background. It is easy to suppress it due to its comparatively small cross section. 


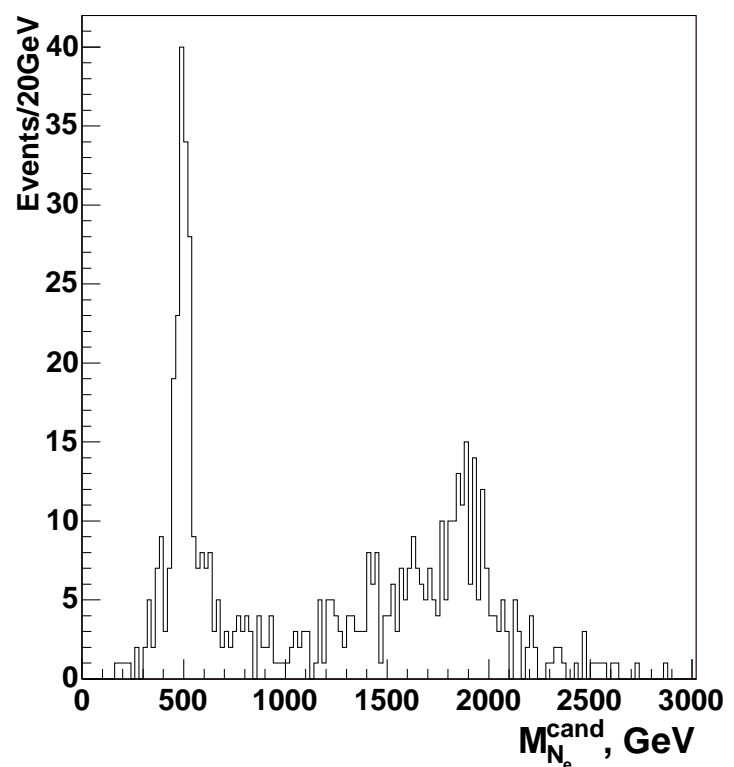

Figure 4: The distribution of the reconstructed invariant mass $M_{N_{e}}^{c a n d}$ for the events simulated with $500 \mathrm{GeV}$ neutrinos. All combinations are shown. The normalization is arbitrary

- The $t \bar{t}$ production turned out to be one of the most important backgrounds. The simulation was made with PYTHIA (standard CMS DST production). In the simulation of this background only leptonic $W$ decay modes were considered. It was checked that other $W$ decay modes do not contribute. The cross section of the $t \bar{t}$ production simulated this way is about two orders of magnitude larger than the signal cross section at LRRP. For the cross section we used the PYTHIA value multiplied by the $\mathrm{K}$ factor corresponding to the total $t \bar{t}$ production cross section (with all decay modes allowed) of $830 \mathrm{pb}$ (NLO value). We tried the alternative way to simulate this important background with the program TOPREX [28]. This program correctly takes into account the spin correlations between the $t$ and $\bar{t}$ and uses the TAUOLA code for $\tau$ decays. The fast simulation showed that the $t \bar{t}$ background obtained this way is not higher than with PYTHIA, so the usage of the PYTHIA production does not underestimate the background.

- another important source of the background is the $Z+$ jet process. This reaction has a cross section which is about 5 orders of magnitude larger than the signal cross section at LRRP. The simulation of these events even at the level of event generator would require a lot of the CPU time. In order to reduce the CPU time the simulation was performed with PYTHIA requiring the minimal allowed $p_{\perp}$ of the hard process to be $20 \mathrm{GeV}$. It was checked with loose cuts that this limit does not change the estimated number of background events for a given luminosity. This background is suppressed by the same cut as the $Z W$ production and by the $P_{t}$ cuts on leptons and jets. In Figure 7 one can see the long tail of the $Z$ invariant mass in this background process and how $M_{l l}$ can be used for its suppression. It is also difficult to make directly a full simulation with sufficient statistics. For this reason only preselected events (less than $10^{-3}$ fraction of all generated events) were fully simulated.

- other possible sources of background are the $Z H$ and $W H$ productions. The cross section of these processes is small and the background is easy to suppress. The suppression of $W H$ process that gives more background events than $Z H$ is illustrated in the last colomn of the background reduction table. The Higgs mass in this simulation was $m_{H}=190 \mathrm{GeV}$.

In Figure 8 the reconstructed $N_{e}$ mass peak in presence of the SM background is shown. The peak is well visible, though the background seems to be rather high in the mass region of several hundred $\mathrm{GeV}$. However, if we require the reconstructed invariant mass of the system $e_{1} e_{2} j_{1} j_{2}$ for the same event to be greater than $1 \mathrm{TeV}$, the background under the $N_{e}$ peak drops dramatically, as illustrated in Figure 9. In Figure 10 the reconstructed $W_{R}$ mass peak in presence of the SM background is shown.

In Figure 11 the plots illustrating the $P t$ balance in the decay of $W_{R}$ are shown. The lower plots, in principle, can 


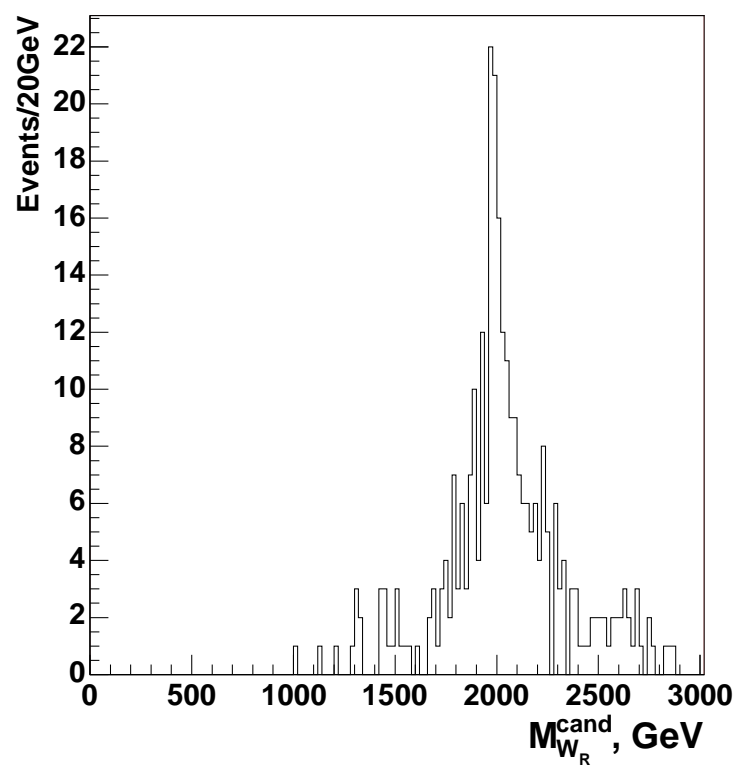

Figure 5: The distribution of the invariant mass $M_{W_{R}}^{c a n d}$ for the reconstructed events simulated with the $W_{R}$ mass of $2000 \mathrm{GeV}$. The normalization is arbitrary.

be used for the rejection of background since the latter tends to have lower pt of the lepton coming directly from $W_{R}$. However, the cut on this variable is less efficient than the $W_{R}$ mass cut.

It is supposed that the search will be performed by scanning the mass spectrum of $N$ candidates after selecting events with $M_{e e j j}>1 T e V$. For the part of parameter space with $M_{W_{R}}<1.5 T e V$ events with $M_{e e j j}>700 G e V$ should be selected. Note that in the background reduction table below the cut $M_{e e j j}>1 T e V$ is shown as the last one, this is done to illustrate better its effect.

In Figure 12 the optimization of the $M_{e j j}$ mass window cut is illustrated. It is applied here as the last cut. In the calculation of efficiency, here and in the background reduction table, we assume that an event passes this cut if at least one of the $e j j$ combinations falls in the mass window. In Figure 13 the distributions of invariant masses in the $\left[M_{e_{1} / e_{2} j_{1} j_{2}}, M_{e_{1} e_{2} j_{1} j_{2}}\right]$ planes reconstructed for the background events are shown ( $e_{1}$ has a highest $\left.E_{t}\right)$. It is seen that the background increases for the $r=M_{N_{e}} / M_{W_{R}}$ values close to unity.

Table 1: Evolution of numbers of the signal and background events versus selection criteria. The initial numbers of background events correspond to the statistics that would be collected at the CMS detector for the integrated luminosity $L_{t}=30 \mathrm{fb}^{-1}$

\begin{tabular}{|c|c|c|c|c|c|}
\hline Step & $\begin{array}{c}\text { Signal } \\
\text { LRRP }\end{array}$ & $t \bar{t}$ & $\mathrm{Z}+$ jet & ZW & WH \\
\hline Initial & 4965 & $2.64 \times 10^{6}$ & $6.2 \times 10^{7}$ & $6 \times 10^{4}$ & 11000 \\
\hline Primary selection & 2782 & $1.5 \times 10^{5}$ & - & 38 & 728 \\
\hline Two isolated leptons & 2332 & 152000 & - & 15 & 165 \\
\hline$M_{l l}$ cut & 2246 & 17200 & 3870 & 0 & 72 \\
\hline$M_{N_{e}}^{c a n d}$ mass window & 970 & 3430 & 1000 & 0 & 2 \\
\hline$M_{N_{e}}^{c a n d}$ mass window $+M_{W_{R}}^{c a n d}>1 \mathrm{TeV}$ & 938 & 198 & 96 & 0 & 0 \\
\hline
\end{tabular}

Due to its nature, the Majorana neutrino can decay either as a Dirac neutrino or as a Dirac anti-neutrino. Since the final states are distinguishable, there is no interference between these two types of the decay channel. Assuming that the $N_{l}$ neutrino couples dominantly to a single light charged lepton of $l$-flavor, then the Dirac neutrinos will always decay into opposite charged leptons $l$, while the Majorana neutrinos will decay like that half of the time, but the other half into like-sign $l$ 's. Like-sign charged leptons could provide a clean signal for the production of 


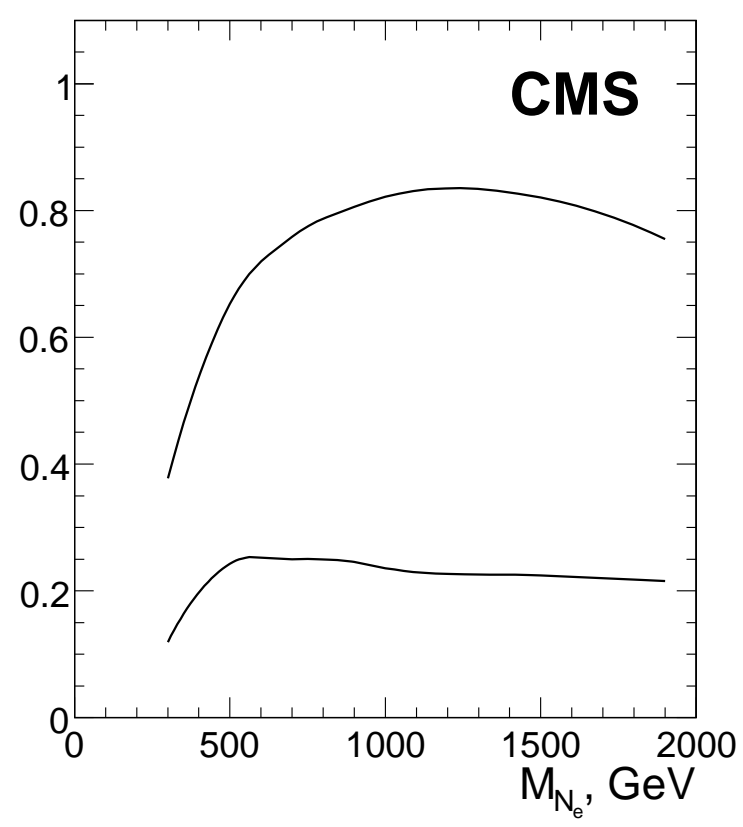

Figure 6: The probability to pass the primary selection for the $p p \rightarrow W_{R} \rightarrow e+N_{e} \rightarrow 2 e+2 j e t$ events as a function of the $N_{e}$ mass for $M_{W_{R}}=2 \mathrm{TeV}$. The lower curve is the probability to have all four $W_{R}$ decay products reconstructed as separate CMS reconstruction objects.

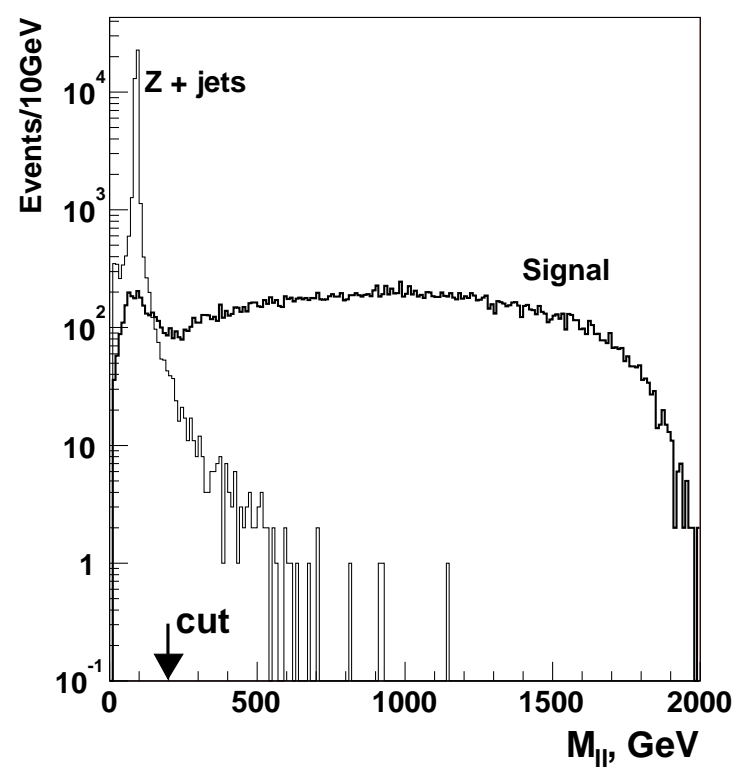

Figure 7: Illustration of the use of the cut $M_{l l}>200 \mathrm{GeV}$ for the suppression of the $Z g$ background. 


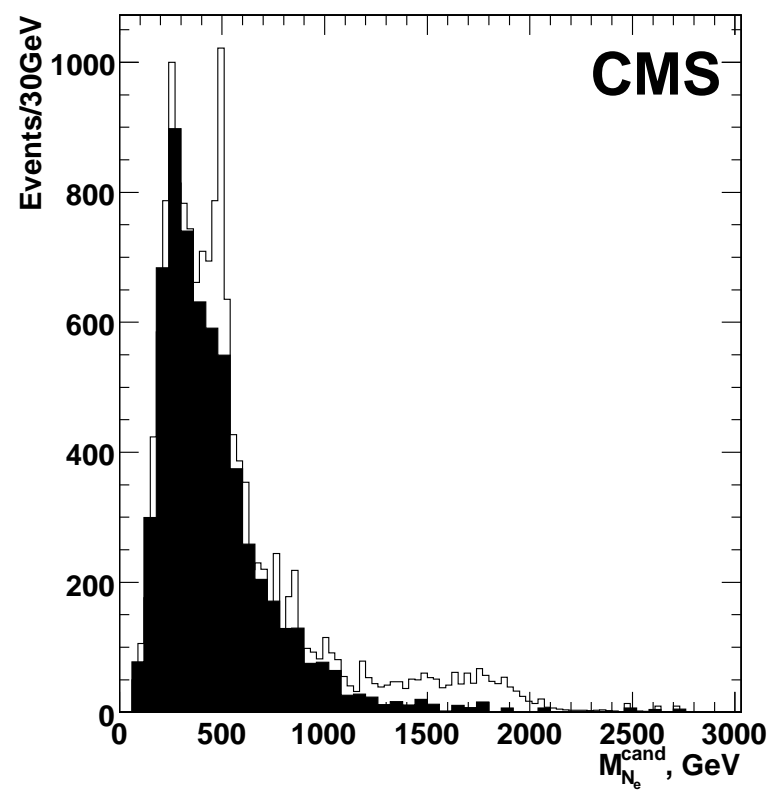

Figure 8: The heavy neutrino mass peak reconstructed together with the SM background (shaded histogram) for an integrated luminosity $L_{t}=30 \mathrm{fb}^{-1}$.

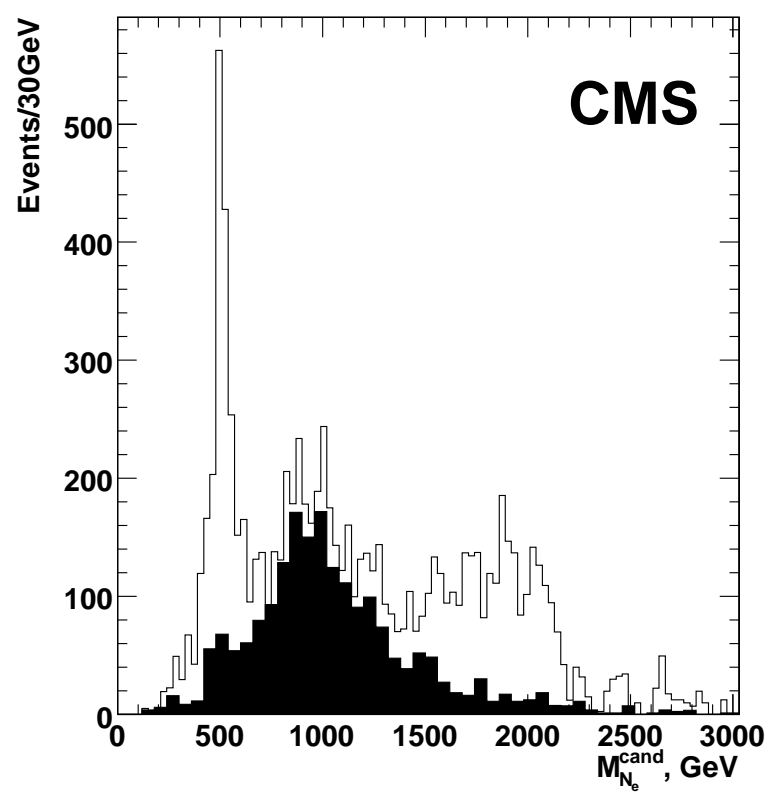

Figure 9: The heavy neutrino mass peak reconstructed together with the SM background (shaded histogram). The invariant mass of the $e_{1} e_{2} j_{1} j_{2}$-system is required to be greater than $1 \mathrm{TeV}$. No mass window cuts are applied. The integrated luminosity is $L_{t}=30 \mathrm{fb}^{-1}$. 


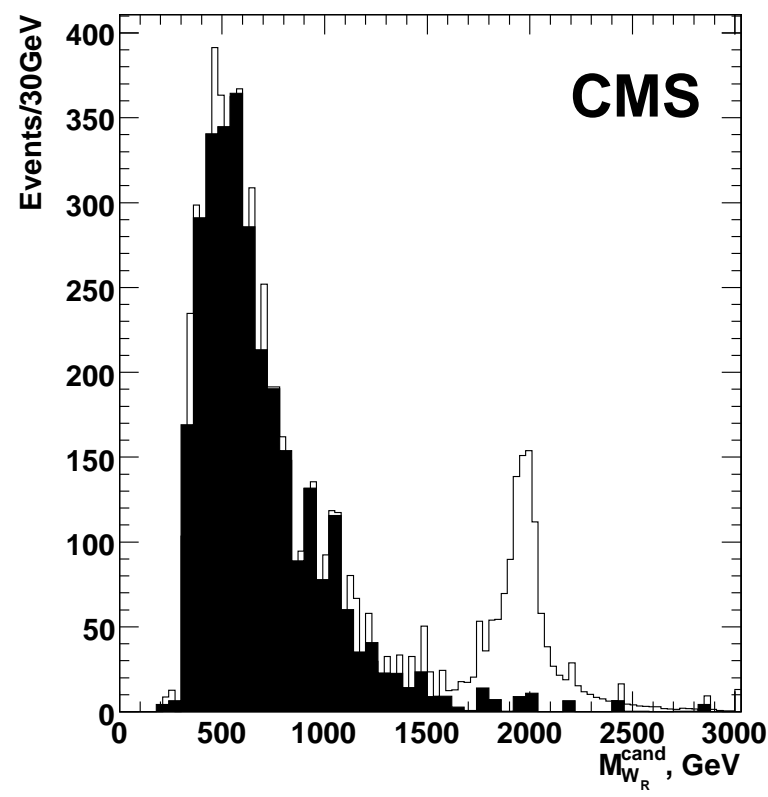

Figure 10: The $W_{R}$ mass peak reconstructed together with the SM background (shaded histogram) for the integrated luminosity $L_{t}=30 \mathrm{fb}^{-1}$.
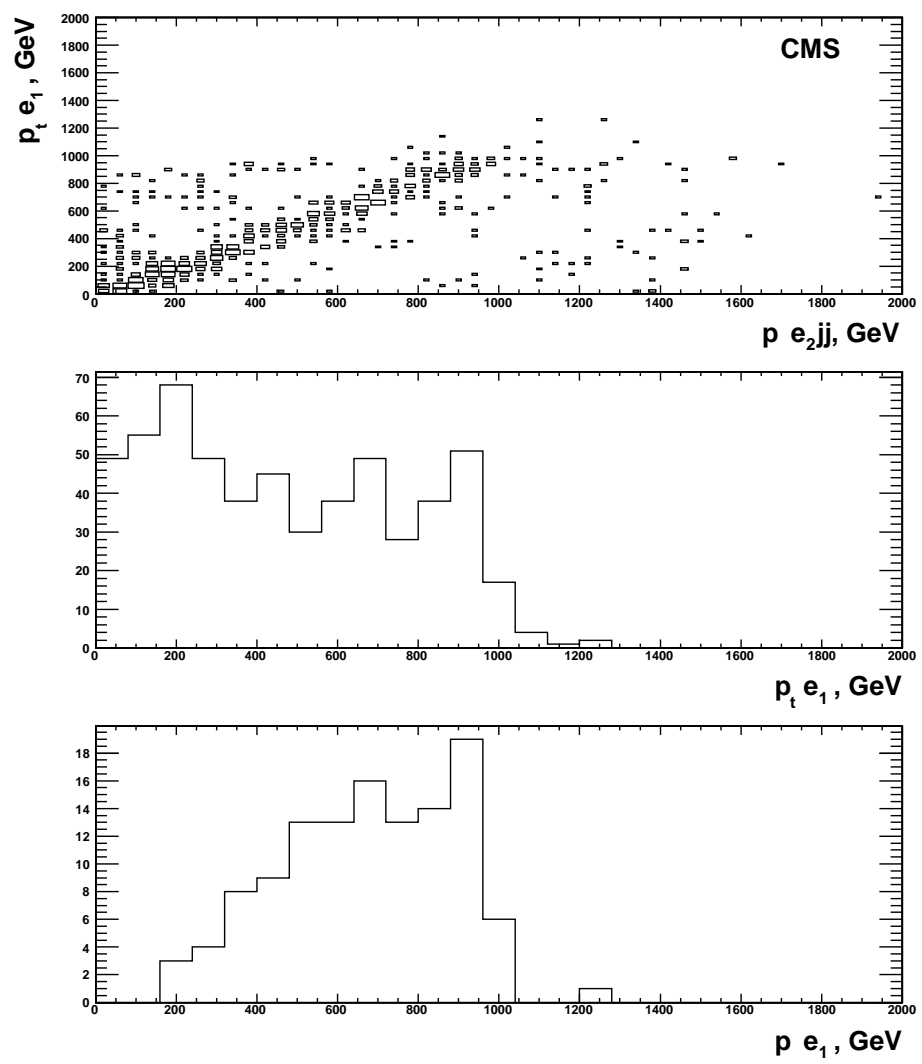

Figure 11: Upper plot: signal, $P_{t}$ of one of the electrons vs total $P_{t}$ of another lepton plus the two chosen jets. Lower plots are the $\mathrm{Y}$ projections of the upper plot for all events and for accepted events 

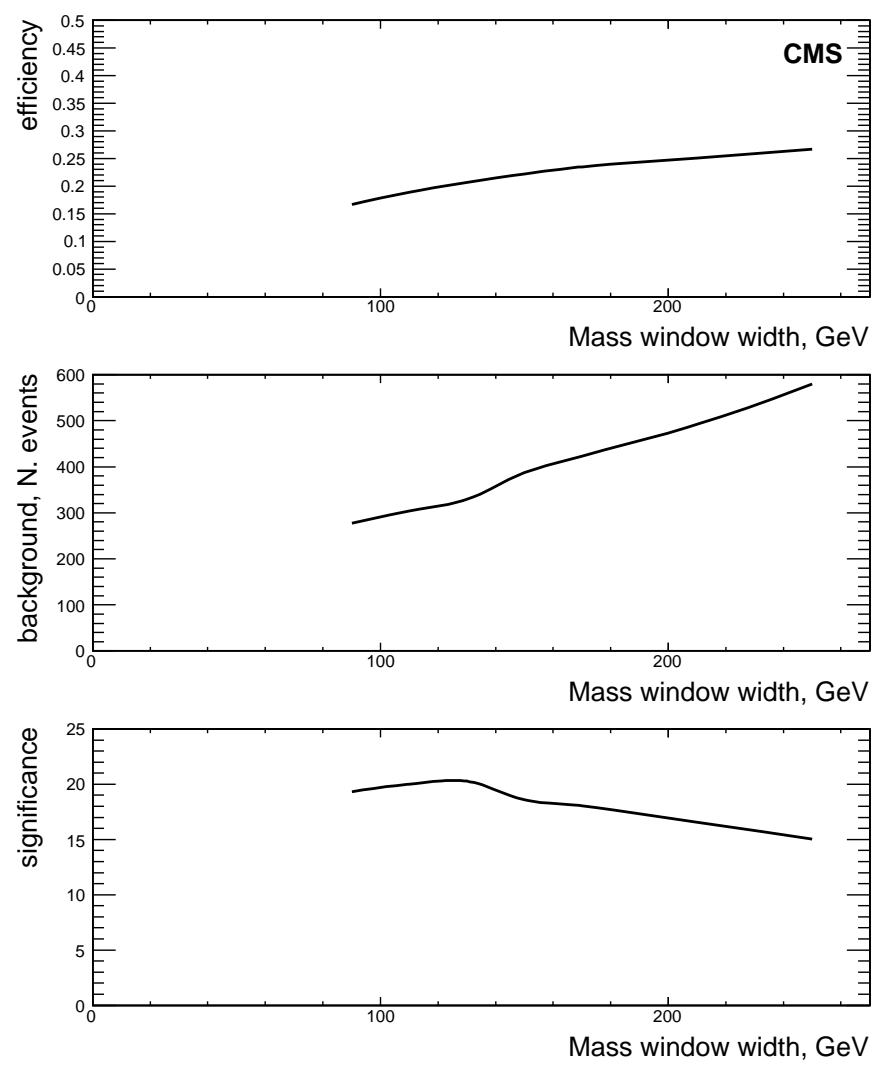

Figure 12: Signal efficiency, remaining background and significance as functions if the $M_{e j j}$ mass window width applied as the last cut. 
Majorana neutrinos in case of $l$ associated with electrons or muons [29]. At the present stage of the analysis it was found that the requirement of two like-sign leptons in the final state does not improve significantly the sensitivity due to the loss of $50 \%$ of the signal events. But it is an excelent cross check in the case of signal observation. The only sources of the like-sign background events are the $Z H$ and $W H$ production. However, the cross sections of these processes are small, thus making the search for heavy neutrino events with the like-sign leptons almost physical background-free.

It was also observed that the more important background for the like-sign analysis is related to the the incorrect reconstruction of charge of electron tracks. We found that such tracks can be effectively suppressed by a lower cut on the number of hits in the track. A cut at 6 hits gives a suppression factor of about 3 keeping the efficiency to tracks with a correct reconstruction at the level of $90 \%$.

\section{Results}
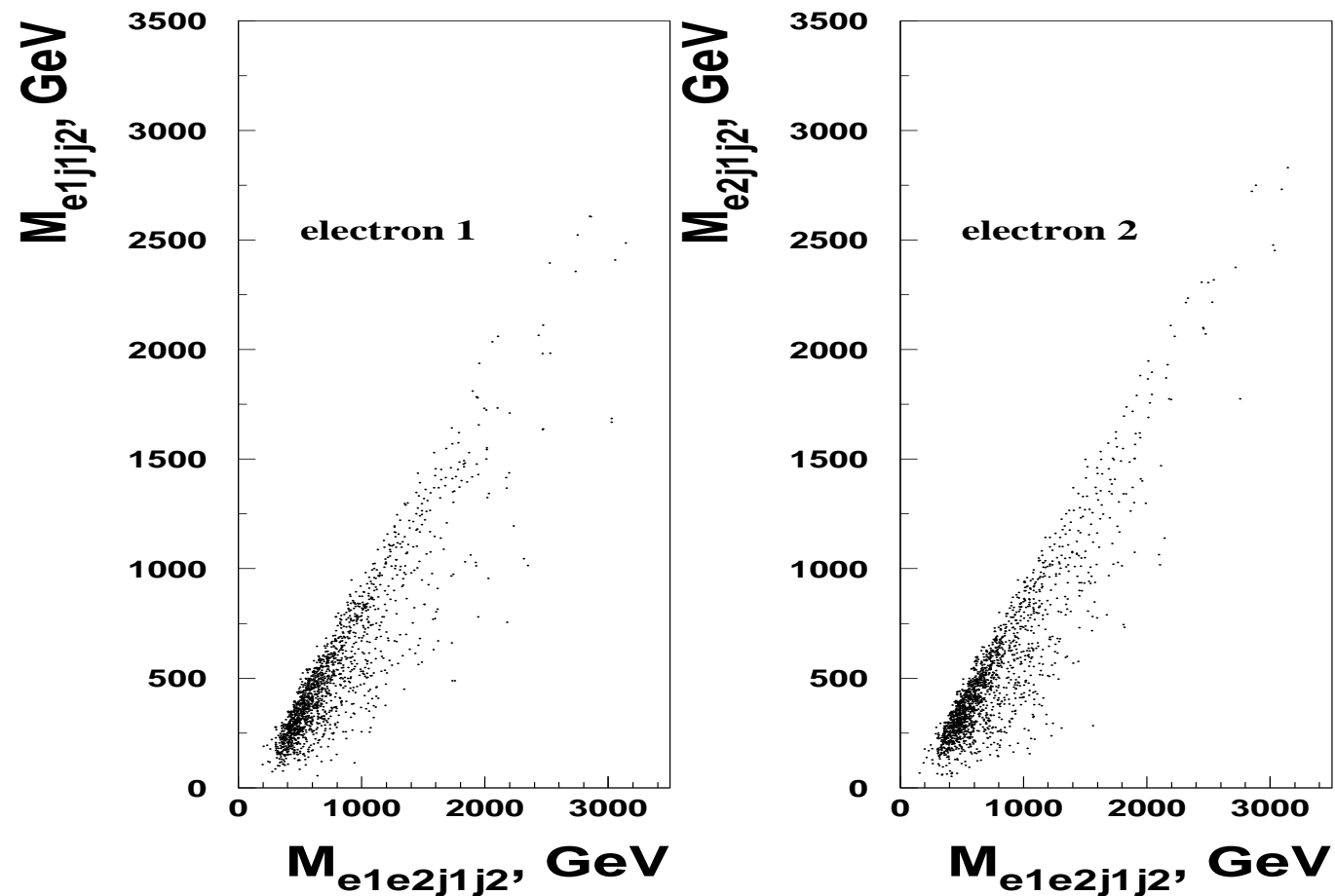

Figure 13: The distribution of invariant masses in the $\left[M_{e_{1} j_{1} j_{2}}, M_{e_{1} e_{2} j_{1} j_{2}}\right]$ (left plot) and $\left[M_{e_{2} j_{1} j_{2}}, M_{e_{1} e_{2} j_{1} j_{2}}\right]$ (right plot) planes, reconstructed for the background events in the CMS detector. The applied cuts are presented in Table 1.

The discovery potential of the events with $W_{R}$ and $N_{e}$ in CMS was calculated using the following relation [30]:

$$
S=2\left(\sqrt{N_{S}+N_{B}}-\sqrt{N_{B}}\right) \geq 5
$$

where $N_{S}$ and $N_{B}$ are the numbers of signal and background events respectively, which pass the selection criteria described in Table 1 for both $M_{e_{1} / e_{2} j_{1} j_{2}}$ and $M_{e_{1} e_{2} j_{1} j_{2}}$ variables.

The corresponding discovery contour in the $\left(M_{W_{R}} ; M_{N_{e}}\right)$ plane is shown in Figure 14. After three years of running at low luminosity $\left(30 \mathrm{fb}^{-1}\right.$ ) the use of $p p \rightarrow W_{R} \rightarrow e N_{e} \rightarrow$ eejj reaction allows to discover $W_{R}$ and $N_{e}$ with masses up to $3.5 \mathrm{TeV}$ and $2.3 \mathrm{TeV}$, respectively. It is interesting that for the LRRP point the $5 \sigma$ discovery requires just a few $\mathrm{fb}^{-1}$ of the integrated luminosity.

The uncertainty of these results coming from various background uncertainties is small since the background itself is rather small and the discovery region is usually limited by the fast drop of the signal cross section at high ratios $r=M_{N_{e}} / M_{W_{R}}$ or by the fast drop of efficiency at small $r$. This is illustrated by the uncertainty on the jets 
energy scale of $3 \%$ [31] that we studied. It leads to uncertainties on the background of $5-10 \%$. This changes the significance by $3-10 \%$. The discovery region upper boundary shifts by $0.5-3 \%$, the lower one by $2-4 \%$.

As to the signal cross section uncertainties, we studied the one from PDF (parton density functions) [32]. By taking different PDF sets we found that the full amplitude of the cross section change is about $6 \%$ in the whole discovery region. No change of acceptance was observed. Taking 6\% pessimistically as the PDF uncertainty, it was estimated from the cross section slopes of Figure 3 that the uncertainty of the upper boundary of the discovery region is $1-2 \%$. The uncertainty of its lower boundary coming from PDF is $2-3 \%$.

\section{Conclusion}

We have studied the CMS potential for the discovery of a heavy right-handed Majorana neutrino $N_{e}$ and a heavy $W_{R}$ gauge boson. The necessary suppression of the SM background processes is achieved by requiring two isolated electrons and at least two jets being reconstructed in the final state with loose constraints on their kinematical variables. For an integrated luminosity of $30 \mathrm{fb}^{-1}$ these new particles can be observed if the masses $M_{W_{R}}$ and $M_{N_{e}}$ are less than 3.5 and $2.3 \mathrm{TeV}$, respectively. For the masses $M_{W_{R}} \simeq 2 \mathrm{TeV}$ and $M_{N_{e}} \simeq 0.5 \mathrm{TeV}$, the $W_{R}$ and $N_{e}$ particles can be discovered already after one month of running at low luminosity.

The sensitivity of CMS to $W_{R}$ and $N_{e}$ at high luminosity of $300 \mathrm{fb}^{-1}$ after three years of running should make the discovery region wider. However, to estimate the corresponding discovery region the effects of the pile-up have to be carefully studied. This work, as well as the the analysis of the effect of the $W_{R}$ polarization, are in progress.

\section{Acknowledgments}

We would like to thank S. Slabospitsky for useful discussions and L.Pape for the critical reading of the manuscript and useful suggestions. We would also like to acknowledge the help of Yu. Andreev and A. Toropin.

This work was supported by the Grant RFBR 04-02-16020. 


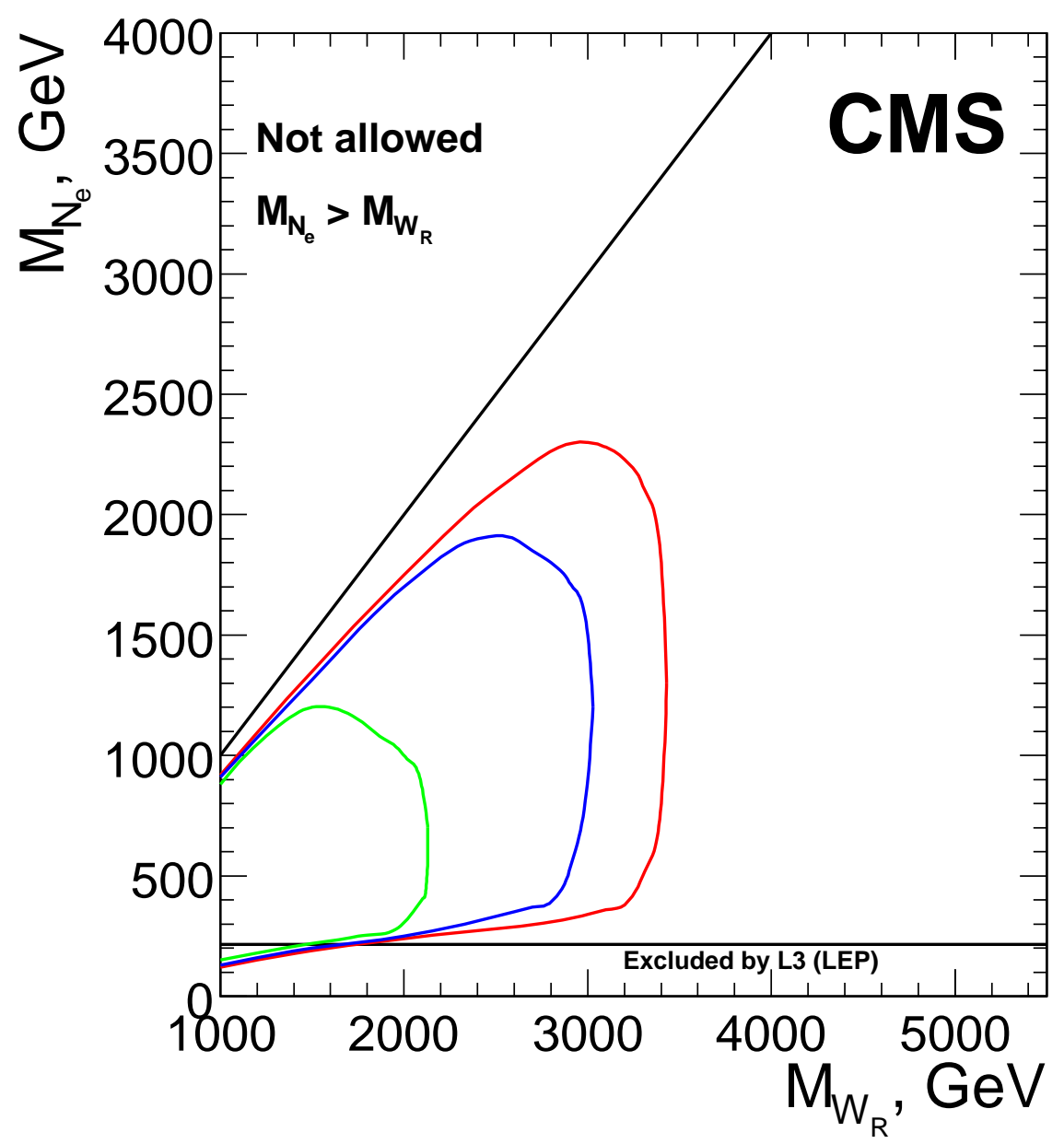

Figure 14: CMS discovery potential of the $W_{R}$ boson and right-handed Majorana neutrinos of the Left-Right Symmetric model for an integrated luminosity $L_{t}=30 \mathrm{fb}^{-1}$ (outer contour) and for integrated luminosities $L_{t}=10 \mathrm{fb}^{-1}, L_{t}=1 \mathrm{fb}^{-1}$ (inner contours). The heavy neutrino masses below the horisontal line are excluded by L3 [27] 


\section{References}

[1] For a recent review, see e.g, N. V. Krasnikov and V. A. Matveev, Search for New Physics at LHC, hep$\mathrm{ph} / 0309200$.

[2] J. C. Pati and A. Salam, Phys. Rev. D 10, 275 (1974);

R. N. Mohapatra and J. C. Pati, Phys. Rev. D 11, 366 (1975);

G. Senjanovic and R. N. Mohapatra, Phys. Rev. D 12, 1502 (1975).

[3] M. Gell-Mann, P. Ramon ans R. Slansky, in Supergravity, ed. P. van Niewenhuizen and D. Freedman (NorthHolland 1979);

T. Yanagida, in Proceedings of the Workshop on the Unified Thoery and the Baryon Number in teh Universe, ed. O. Sawaga and A. Sugamoto (Tsukuba 1979);

R.N. Mohapatra and G. Senjanovic', Phys. Rev. Lett. 44, 912, (1980).

[4] C. Giunti and M. Laveder, Neutrino mixing, hep-ph/0310238.

[5] Ho Tso-hsiu, Ching Cheng-rui, and tao Zhi-jian, Phys. Rev. D 42, 2265, (1990).

[6] A. Data, M. Guchait and D. P. Roy, Phys. Rev. D 47 (1993) 961.

[7] O. Panella, M. Cannoni, C. Carimalo, and Y. N. Srivastava, Phys. Rev. D 65, 035005, (2002).

[8] B. Mukhopadhyaya, Phys. Rev. D49(1994)1350; D. Choudhury, R. Godbole and P. Roy, hep-ph/9211240; A. Ali, A.V. Borisov and N.B. Zamorin, hep-ph/0104123.

[9] A. Ferrari et al., Phys. Rev. D 62, 031001, (2002).

[10] S.N. Gninenko, Phys. Lett. B 427 (1998) 206.

[11] M. Czakon, J.Gluza, and M. Zralek, Phys. Rev. D 59 (1998) 013010.

[12] M. Frank, Phys. Rev. D 60 (1999) 093005.

[13] S.N. Gninenko and N.V. Krasnikov, Preprint CERN-EP-99-38, Aug 1998. 11pp; Phys. Lett. B 450 (1999) 165.

[14] S.N. Gninenko, M.M. Kirsanov, N.V. Krasnikov and V.A. Matveev, hep-ph/0301140, to appear in Yad. Fiz.

[15] J. Polak and M. Zralek, Nucl. Phys. B 363 (1991) 385; A. Pilaftsis, Phys. Rev. D 52 (1995) 459; M. Czakon, J.Gluza, and M. Zralek, Phys. Lett. B 458 (1999) 355.

[16] S. Eidelman et al., Review of Particle Physics, Phys. Lett. B592 (2004) 1.

[17] C.A. Heusch, Nucl. Phys. B 416 (1994) 3.

[18] S.N. Gninenko, M.M. Kirsanov, N.V. Krasnikov and V.A. Matveev, Mod. Phys. Lett. A 17 (2002) 1407.

[19] G. Bell, M. Bander, and A. Soni, Phys. Rev. Lett. 48, (1982) 848; G. Barenboim, J. Bernabeu, J. Prades and M. Raidal, Phys. Rev. D 55, (1997) 4213.

[20] P. Langacker and S. Uma Sankar, Phys. Rev. D 40 (1989) 1569.

[21] S. Abachi et al., D0 Collaboration, Phys. Rev. Lett. 76, (1996) 3271.

[22] T. Rizzo, Phys. Rev. D 50 (1994) 325.

[23] E. Nardi, E. Roulet, and D. Tommasini, Phys. Lett. B 344 (1995) 225; J. Gluza et al., Phys. Lett. B 407 (1997) 45; A. Pilaftsis, Int. J. Mod. Phys. A 14 (1999) 1811.

[24] G. Barenboim and M. Raidal, Nucl. Phys. B 484 (1997) 63 and references therein.

[25] T. Sjöstrand, Comput. Phys. Commun. 82 (1994) 74; T. Sjöstrand, Computer Physics Commun. 39(1986)347; H.U.Bengtsson and T.Sjöstrand, JETSET, Computer Physics Commun.43(1987)367.

[26] J. Botts et al., Phys. Lett. B304, 159, (1993). 
[27] L3 Collaboration, P.Achard et al., Phys. Lett. B 517 (2001) 67.

[28] S.R. Slabospitsky and L. Sonnenschein, "TopRex generator (version 3.25): Short manual”, Comput.Phys.Comm. 148 (2002) 87; hep-ph/0201292.

[29] E. Ma and J. Pantaleone, Phys. Rev. D 40 (1989) 2172.

[30] S.I.Bityukov and N.V.Krasnikov, CMS CR 2002/05, hep-ph/0204326;

S.I.Bityukov and N.V.Krasnikov, Mod. Phys. Lett A13 (1998) 3235.

[31] J.D'Hondt et al., Light quark jet energy scale calibration using the $\mathrm{W}$ mass constraint in single-leptonic $t \bar{t}$ events, CMS Note 2006/025

[32] P.Bartalini, R.Chierci and A. De Roeck, Guidelines for the estimation of theoretical uncertainties at the LHC, CMS Note 2005/013 